\title{
Utility of $\mathbf{R}_{2}{ }^{*}$ Obtained from $\mathrm{T}_{2}{ }^{*}$-Weighted Imaging in Differentiating Hepatocellular Carcinomas from Cavernous Hemangiomas of the Liver
}

\author{
Meiyu Sun ${ }^{1}$, Sheng Wang ${ }^{1,2}$, Qingwei Song ${ }^{1}$, Zhiyuan Wang ${ }^{3}$, Heqing Wang ${ }^{1}$, Dianxiu Ning ${ }^{1}$, Bin $X^{1}{ }^{1}$, \\ Qiang Wei ${ }^{1}$, Ailian Liu ${ }^{1 *}$
}

1 Department of Radiology, the First Affiliated Hospital, Dalian Medical University, Dalian, Liaoning, China, 2 Department of CT and MRI, Fuxin Mineral Hospital, Fuxin, Liaoning, China, 3 Department of Ultrasound, Hunan Provincial Tumor Hospital and Affiliated Tumor Hospital of Xiangya Medical School, Central South University, Changsha, Hunan, China

\begin{abstract}
Purpose: To evaluate the feasibility of applying $\mathrm{R}_{2}{ }^{*}$ values to differentiate hepatocellular carcinomas (HCC) from cavernous hemangiomas of the liver $(\mathrm{CHL})$.

Materials and Methods: This retrospective study was approved by the participating Institutional Review Board and written informed consent for all subjects were obtained. Seventy-three patients with 79 pathologically identified HCCs and 65 patients with 91 clinically or pathologically identified CHLs were enrolled in this study. All subjects underwent a breath-hold multi-echo $\mathrm{T}_{2}{ }^{*}$ weighted MR imaging on a $1.5 \mathrm{~T}$ clinical MR scanner. $\mathrm{R}_{2}{ }^{*}$ values from HCC and CHL groups were compared using the Mann-Whitney non-parametric $U$ test. A cut-off value of $R_{2}{ }^{*}$ was evaluated with receiver operator characteristic (ROC) analysis.

Results: The mean $\mathrm{R}_{2}{ }^{*}$ value was $23.32 \pm 12.23 \mathrm{~Hz}$ (95\% confidence interval [Cl]: $20.58 \mathrm{~Hz}, 26.06 \mathrm{~Hz}$ ) for the HCC group, and $3.66 \pm 2.37 \mathrm{~Hz}(95 \% \mathrm{Cl}: 3.17 \mathrm{~Hz}, 4.15 \mathrm{~Hz})$ for the $\mathrm{CHL}$ group. The mean $\mathrm{R}_{2}{ }^{*}$ value for HCC was significantly higher than that of $\mathrm{CHL}(p<0.001)$. A threshold of $9.48 \mathrm{~Hz}$ for the minimum $\mathrm{R}_{2}{ }^{*}$ value in the diagnosis of HCC resulted in a sensitivity of $96.20 \%$ (76 out of 79 patients), and a specificity of $97.80 \%$ ( 89 out of 91 patients). The positive predictive value (PPV), negative predictive value (NPV) and diagnostic accuracy for HCC were $97.44 \%$ (76 out of 78 patients), $96.74 \%$ (89 out of 92 patients) and $97.06 \%$ ( 165 out of 170 patients), respectively. The AUC for differentiation between these two groups was 0.994 (95\% Cl: $0.980,1.000)$.
\end{abstract}

Conclusions: $\mathrm{R}_{2}{ }^{*}$ is a significant MRI biomarker to differentiate HCC from CHL with satisfying sensitivity and specificity.

Citation: Sun M, Wang S, Song $\mathrm{Q}$, Wang Z, Wang $\mathrm{H}$, et al. (2014) Utility of $\mathrm{R}_{2}{ }^{*}$ Obtained from $\mathrm{T}_{2}{ }^{*}$-Weighted Imaging in Differentiating Hepatocellular Carcinomas from Cavernous Hemangiomas of the Liver. PLoS ONE 9(3): e91751. doi:10.1371/journal.pone.0091751

Editor: Jiani Hu, Wayne State University, United States of America

Received October 27, 2013; Accepted February 15, 2014; Published March 14, 2014

Copyright: $\odot 2014$ Sun et al. This is an open-access article distributed under the terms of the Creative Commons Attribution License, which permits unrestricted use, distribution, and reproduction in any medium, provided the original author and source are credited.

Funding: These authors have no support or funding to report.

Competing Interests: The authors have declared that no competing interests exist.

*E-mail: liuailian_dalianmu@126.com

\section{Introduction}

Hepatocellular carcinoma (HCG) is the most common type of primary malignant hepatic tumor, which is the third leading cause of cancer-related death worldwide [1]. Cavernous hemangioma of the liver (CHL) is the most frequently diagnosed benign hepatic tumor, developing in $0.4-20 \%$ of the population [2]. Treatment for CHL is markedly different than HCG [3,4]. Characterization of CHL and HCC using ultrasonography (US) and contrastenhanced computed tomography (CT) is well established and routinely applied in liver lesion diagnosis [5-7]. Both qualitative and quantitative magnetic resonance imaging (MRI) methods are also used for the diagnosis of liver diseases [8-13]. Subjective qualitative analysis depends on interpreters' experience and expertise, thereby potentially limiting its diagnostic accuracy and reproducibility. Additionally, administration of gadolinium contrast agents is required for this type of subjective interpretation.
Several noninvasive, quantitative MR methods for differentiating malignant tumors from benign hepatic lesions have also been proposed, including calculated $\mathrm{T}_{2}$ relaxation times [9-11] and apparent diffusion coefficient (ADC) derived from diffusion weighted imaging [13]. Despite the availability of these noninvasive methods mentioned above, the considerable overlaps, sequence dependent results, and long acquisition times have prevented their wide use in routine clinical applications. Therefore, to circumvent these limitations, the current study proposes the establishment of an accurate, fast and robust quantitative method using non-invasive MRI.

$\mathrm{R}_{2} *\left(1 / \mathrm{T}_{2} *\right.$ in $\left.1 / \mathrm{s}\right)$ is sensitive to both micro and macro vasculature due to susceptibility alteration [14]. A prior study has already demonstrated the feasibility of using $\mathrm{R}_{2} *$ values to predict the characteristic micro vascular invasion of HCC using a blood oxygen level-dependent (BOLD) test [14]. Several recent studies have also suggested that multi-echo $\mathrm{T}_{2}{ }^{*}$-weighted imaging might 


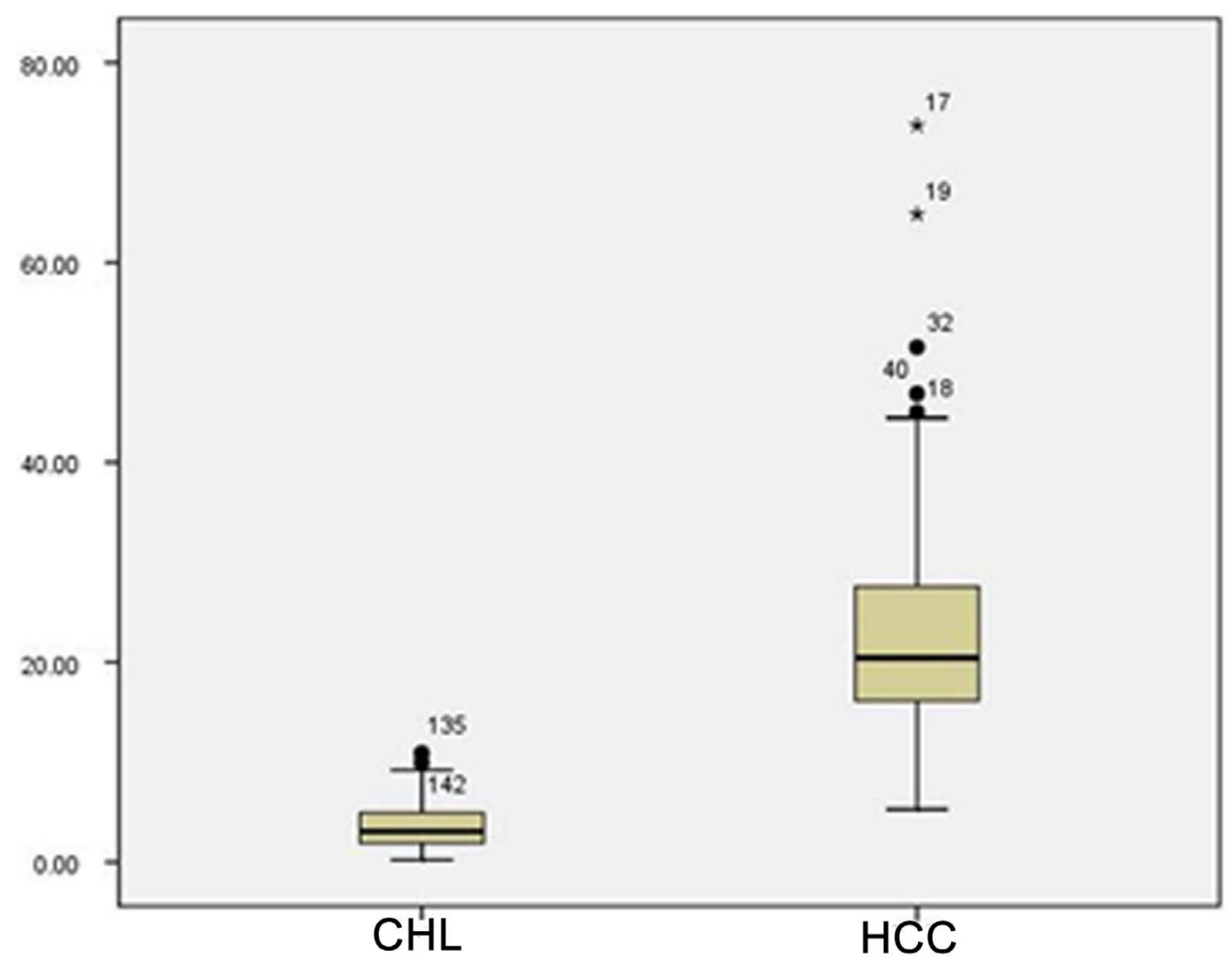

Figure 1. Box plots of $\mathbf{R}_{\mathbf{2}}$ * value of HCCs and CHLs. $\mathrm{R}_{2}{ }^{*}$ value is differed significantly between HCCs and CHLs. Boxes stretch across interquartile range (IR), from lower quartile (Q1) to upper quartile (Q2); whiskers show the smallest data point that is greater than [Q1-1.5IR] and the largest data point that is smaller than [Q2+1.5IR]. The horizontal line through each box represents the median value. The dot indicates any outlier with its code number.

doi:10.1371/journal.pone.0091751.g001

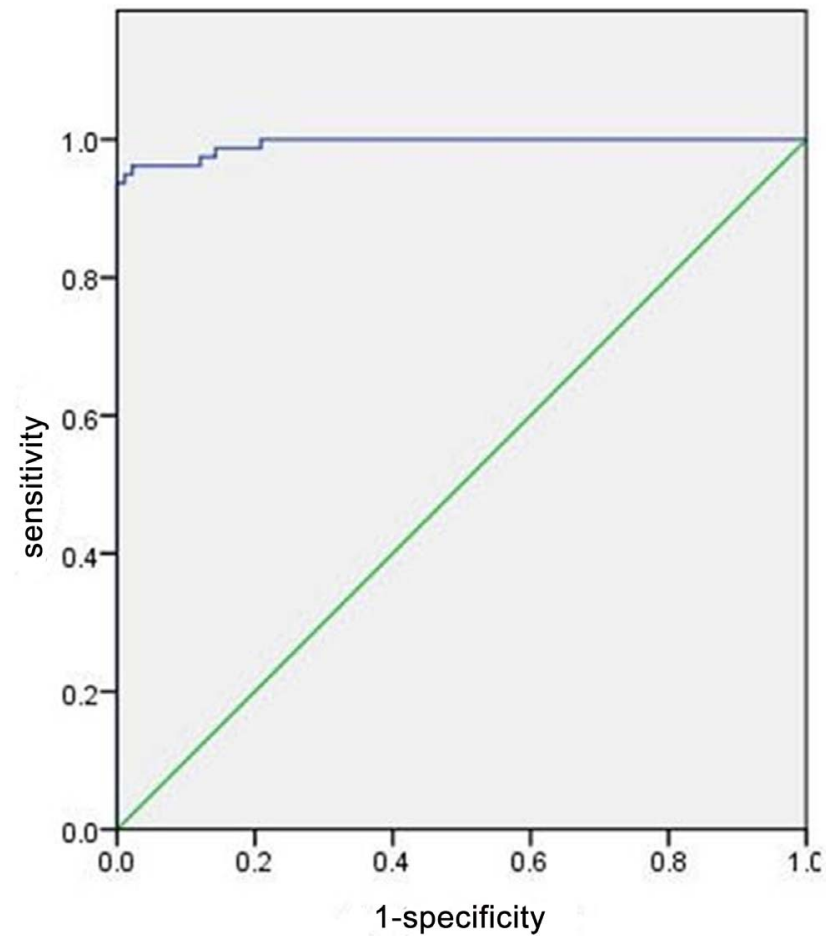

Figure 2. ROC curve for the diagnosis of HCCs. Area under ROC curve is 0.994 ( $95 \% \mathrm{Cl}: 0.980,1.000)$.

doi:10.1371/journal.pone.0091751.g002 have the potential to identify HCC [15-17]. However, in current literature, there is no study focused on the usefulness of $\mathrm{R}_{2} *$ in differentiating HCG from CHL. Therefore, the purpose of this study is to investigate the feasibility of using $\mathrm{R}_{2} *$ values from multiecho $\mathrm{T}_{2}{ }^{*}$-weighted imaging to differentiate HCG and CHL.

\section{Materials and Methods}

\section{Patients}

This study was approved by the Institutional Review Board of the First Affiliated Hospital of Dalian Medical University and all subjects signed a written informed consent. From November 2011 to February 2013, 138 consecutive HCC and CHL patients were enrolled in this study. The study excluded HCC patients $(n=465)$ with prior treatment including antineoplastic chemotherapy, transarterial chemoembolization, radiofrequency ablation, or chemo radiation. An additional $14 \mathrm{HCC}$ and $12 \mathrm{CHL}$ patients were excluded due to severe motion artifact from an unsuccessful breath-hold or cardiac and aortic pulsation. Seventy-three patients (mean age $=60.10$ years $\pm 11.32(\mathrm{SD}) ; 60 \mathrm{M}$ : $13 \mathrm{~F})$ with 79 pathologically identified $\mathrm{HCC}$ and 65 patients (mean age $=47.74$ years $\pm 9.03(\mathrm{SD})$; $26 \mathrm{M}$ : $39 \mathrm{~F}$ ) with 91 clinically or pathologically identified CHL were enrolled in this study. None of the subjects enrolled had hemochromatosis, severe hepatic steatosis, or intravenous administration of super-paramagnetic iron oxide particles before the MRI examination.

For the 73 subjects with HCGs (79 lesions), all had histopathological confirmation after biopsy $(n=15$, including two patients with two lesions) or surgical resection $(\mathrm{n}=58$, including 57 patients with solitary HCC lesion and one patient with five HCC nodules). 
The interval between the date of the MRI study and sample collection for histopathological confirmation ranged from 4-7 days (average 5.5 days $\pm 1.0(\mathrm{SD})$ ). Among the 65 patients with CHL, 26 patients carried diagnosed cavernous hemangiomas for more than two years before the MR study and had no changes in morphology and size during this period. The diagnosis of CHL was established by means of observed hyper intensity on $\mathrm{T}_{2}$-weighted images and slightly irregular or globular peripheral enhancement with gradual filling in the center of the lesion on delayed images in CT and/or MRI with intravenous administration of contrast material [18]. At the time they had their MR examinations, confirmed diagnosis of CHL for another 37 patients was made based on typical patterns of enhancement on dynamic MRI examination and the absence of change in morphology and size of the lesions during a follow-up period of more than six months after the imaging studies (mean: 12 months; range: 6-16 months). The remaining two patients with CHL had histopathological confirmation after hepatectomy.

\section{MR Imaging}

Each patient was instructed to hold his or her breath in a fixed position on all breath-hold sequences. All examinations were performed on a $1.5 \mathrm{~T}(40 \mathrm{mT} / \mathrm{m}) \mathrm{MR}$ scanner (Signa, HDxt, General Electric [GE] healthcare, USA) with an 8-channel phased array coil, adequately positioned to cover the upper abdomen of the patient lying in the supine position. All patients underwent a routine MRI examination initially for the upper abdomen which included transverse $\mathrm{T}_{2}$-weighted imaging and dynamic $\mathrm{T}_{1^{-}}$ weighted imaging. $\mathrm{T}_{2}$-weighted fat-suppressed images were

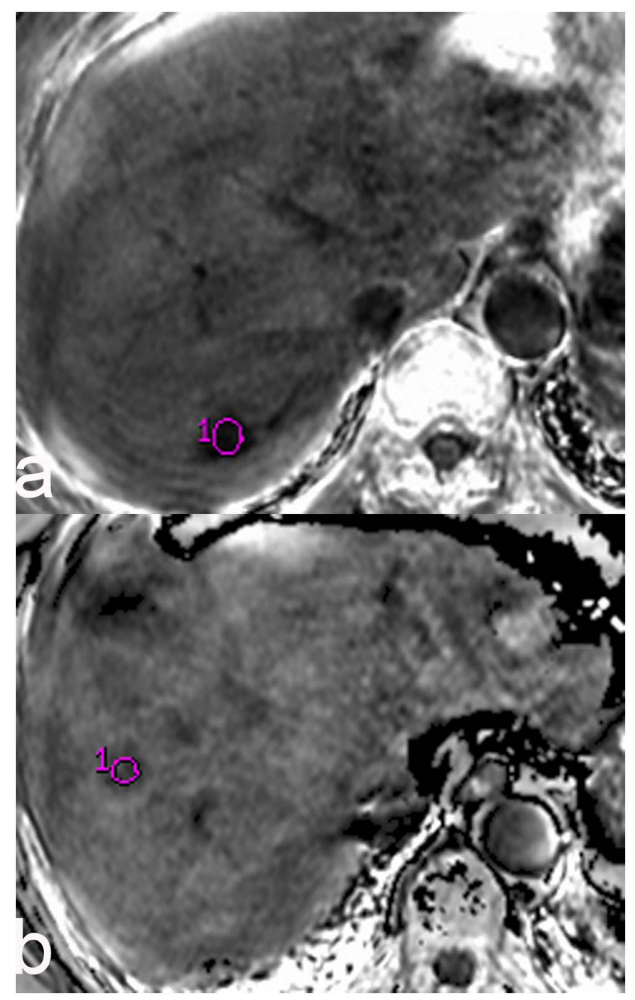

Figure 3. $\mathbf{R}_{\mathbf{2}}$ * images of $\mathbf{C H L}$ and HCC. a. $A$ CHL $\mathrm{R}_{2}{ }^{*}$ image in a 51year-old female. Transverse $R_{2}{ }^{*}$ map shows a well-marginated lesion at segment VII of the liver with a mean $R_{2}{ }^{*}$ value of $7.77 \mathrm{~Hz}$. b. A HCC $R_{2}{ }^{*}$ image in a 59-year-old female. Transverse $R_{2}{ }^{*}$ map shows a wellmarginated lesion at segment VIII of the liver with a mean $R_{2}{ }^{*}$ value of $14.41 \mathrm{~Hz}$.

doi:10.1371/journal.pone.0091751.g003 obtained with a respiratory-triggered fast spin echo ( $\mathrm{T}_{2}$ WFSE) sequence. The parameters were: repetition time $(\mathrm{TR})=6000 \mathrm{~ms}$, echo time $(\mathrm{TE})=102 \mathrm{~ms}$, bandwidth $=62.5 \mathrm{~Hz} /$ pixel, $\mathrm{NEX}=3$, acquisition matrix $=288 \times 224$, field of view $(\mathrm{FOV})=380 \mathrm{~mm} \times 380 \mathrm{~mm}$, slice thickness $=6 \mathrm{~mm}$, gap $=1.5 \mathrm{~mm}$, and scan time three minutes. Dynamic $\mathrm{T}_{1}$ weighted MRI was obtained by using a 3D T1-weighted fatsuppressed spoiled gradient echo sequence utilizing the manufacturer's liver acquisition volume acceleration (LAVA) technique before and after injection of gadopentetate dimeglumine (Magnevist, Schering, Guangzhou, China) at a dose of $0.1 \mathrm{mmol}$ per kilogram of body weight mass in the distal part of a connecting line into an antecubital vein at a rate of $2 \mathrm{ml} / \mathrm{s}$ using a power injector (Meorao, Spectris, USA). Acquisition parameters were as follows: $\mathrm{TR}=3.7 \mathrm{~ms}, \mathrm{TE}=1.8 \mathrm{~ms}$, inversion time $=7 \mathrm{~ms}$, flip angle $=20^{\circ}$, parallel imaging (ASSET: array spatial sensitivity encoding technique, GE) acceleration factor $=2$, bandwidth $=62.5 \mathrm{~Hz}$ / pixel, $\quad \mathrm{NEX}=0.7, \quad$ acquisition $\quad$ matrix $=272 \times 180$, FOV $=380 \mathrm{~mm} \times 380 \mathrm{~mm}$, slice thickness $=4.4 \mathrm{~mm}$, gap $=-$ $2.2 \mathrm{~mm}$, and scan time was about $17 \mathrm{~s}$ per phase. Multi-echo $\mathrm{T}_{2}{ }^{*}$-weighted was performed prior to dynamic $\mathrm{T}_{1}$-weighted imaging, the parameters were: $\mathrm{TR}=16.4 \mathrm{~ms}$; $\mathrm{TE}=2.1,5.0,7.9$, $10.9,13.8 \mathrm{~ms}$; flip angle $=20^{\circ}$; ASSET acceleration factor $=2$ : bandwidth $=62.5 \mathrm{~Hz} /$ pixel; $\quad \mathrm{NEX}=0.67$; acquisition matrix $=256 \times 192 ; \quad$ FOV $=380 \mathrm{~mm} \times 380 \mathrm{~mm}$; slice thickness $=2 \mathrm{~mm}$; and $21 \mathrm{~s}$ scan time for one breath-hold acquisition.

\section{MRI Evaluation}

Two radiologists (Liu AL with 20 years and Sun MY with 10 years of experience in reading hepatic MRI images) reviewed the MRI images using a PACS work station (Daijia healthcare, Shanghai, China). They were both blinded to the original MRI reports and did not know the proportion of each type of lesion in the study population. MRI diagnosis of each patient was obtained in conference by two observers informed with necessary clinical history and previous images.

\section{$\mathrm{R}_{2}^{*}$ Measurement}

The multi-echo $\mathrm{T}_{2}{ }^{*}$-weighed images were transferred to an ADW4.4 workstation (Sun Microsystems, Santa Clara, Calif, USA) for post-processing. To generate the $\mathrm{R}_{2}{ }^{*}$ map, the logarithms of the pixel values, $\mathrm{S}$, were fitted to a linear function using regression analysis, according to the equation $\mathrm{S}(\mathrm{TE})=$ $\mathrm{S}(0) \cdot \exp \left(-\mathrm{TE} \cdot \mathrm{R}_{2} *\right)$. All measurements were performed by a radiologist (WS with 5 years of experience in reading liver MRI images) who was blinded to the diagnosis of the subjects. The largest possible ROI, which represented the signal intensity of the tumor, was manually outlined in a homogeneous area on the $\mathrm{R}_{2}$ * map. The contrast and morphologic characteristics in the three phases of contrast-enhanced $\mathrm{T}_{1}$-weighted imaging and $\mathrm{T}_{2}$ weighted imaging were used to guide ROI placement in order to avoid visible vasculature, biliary structures, hemorrhage, suspected calcification and necrosis. The areas which were severely affected by artifacts caused by the tissue-air interface, pulsations of the heart, or abdominal aorta were not included. To demonstrate the reproducibility of the results, all HCG and CHL lesions were measured three times with the same method by varying placement of the ROIs and the final $R_{2} *$ were taken as the averaged value for the three times. Furthermore, the $\mathrm{R}_{2} *$ values were measured repeatedly on two sessions with a one month interval by the abovementioned observer working in consensus to test the intraobserver concordance. The $\mathrm{R}_{2} *$ value and associated $\mathrm{SDs}$ for each region of interest were calculated on a pixel-by-pixel basis. 
Table 1. $R_{2}^{*}$ value of $H C C$ and $C H L$ groups.

\begin{tabular}{|c|c|c|c|c|c|}
\hline $\mathbf{R}_{\mathbf{2}}{ }^{*}(\mathrm{~Hz})$ & mean $\pm S D$ & $\operatorname{Median}\left(q_{1} ; q_{3}\right)$ & Range & $\mathbf{z}$ & $P$ \\
\hline $\mathrm{HCC}$ & $23.32 \pm 12.23$ & $20.41(16.05 ; 27.59)$ & $5.29-73.74$ & -11.807 & 0.000 \\
\hline $\mathrm{CHL}$ & $3.66 \pm 2.37$ & $3.05(1.78 ; 4.98)$ & $0.22-10.88$ & -11.807 & 0.000 \\
\hline
\end{tabular}

\section{Statistical Analysis}

SPSS 17.0 software for Windows (SPSS Inc., Chicago, Illinois, USA) was used for statistical analysis. The size difference of the HCC and CHL groups were searched for using the student's $t$-test. Mean $\mathrm{R}_{2} *$ values as well as standard deviations, medians, and ranges were calculated for the HCG and CHL groups. The precision of $\mathrm{R}_{2} *$ measurements were tested by the reliability coefficient $\left(r_{c}\right)$. The $r_{c}$ values larger than 0.85 , between 0.50 and 0.85 , and less than 0.50 indicated good concordance, moderate concordance, and poor concordance, respectively. The distributions of $\mathrm{R}_{2}$ * obtained for HCGs and CHLs were displayed in box plots. The Mann-Whitney non-parametric $U$-test was used to compare the mean $\mathrm{R}_{2}{ }^{*}$ value in the $\mathrm{HCC}$ and $\mathrm{CHL}$ groups. A probability value $(p)$ less than 0.05 was considered statistically significant. Receive operating characteristic (ROC) curve analysis was performed to identify the optimum cutoff value which maximized the average of sensitivity and specificity, with HCC defined as test positive and CHL as test negative. Sensitivity, specificity, positive predictive value (PPV), negative predictive value (NPV) and accuracy of diagnosis were subsequently calculated and recorded. The diagnosis performance of $\mathrm{R}_{2} *$ values for differentiating HCGs from CHLs was evaluated with the calculation of the area under the curve (AUC).

\section{Results}

\section{Intraobserver Concordance of the $\mathrm{R}_{2}{ }^{*}$ Value Measurements}

In this cohort, the mean $\mathrm{R}_{2} *$ value of the $\mathrm{HCC}$ group was $23.32 \pm 12.23 \mathrm{~Hz}$ (range, 5.29-73.74) for initial measurements and $21.78 \pm 11.25 \mathrm{~Hz}$ (range, 5.16-72.93) for the repeated measurements. The resulting $r_{c}$ value was 0.93 , which was considered acceptable. The mean $\mathrm{R}_{2}{ }^{*}$ value of the $\mathrm{CHL}$ group was $3.66 \pm 2.37 \mathrm{~Hz}$ (range, $0.22-10.88$ ) for initial measurements and $3.69 \pm 2.25 \mathrm{~Hz}$ (range, 0.21-10.97) for the repeated measurements, resulting in an $r_{c}$ value equal to 0.96 which was acceptable as well. Therefore, the intraobserver concordance of the $\mathrm{R}_{2} *$ value measurements was proven sufficient and the first measurements were used as the final $\mathrm{R}_{2}{ }^{*}$ values.

\section{Comparison of $\mathrm{R}_{2}{ }^{*}$ Values between $\mathrm{HCC}$ and $\mathrm{CHL}$ Groups}

A total of 138 subjects (170 lesions) were enrolled, with a mean lesion size of $3.78 \pm 2.10 \mathrm{~cm}$ (range from $0.89 \mathrm{~cm}$ to $9.34 \mathrm{~cm}$ ). For the $79 \mathrm{HCC}$ lesions, the mean size was $4.26 \pm 2.10 \mathrm{~cm}$ (range from $1.1 \mathrm{~cm}$ to $9.1 \mathrm{~cm}$ ), and the mean $\mathrm{R}_{2}$ * value was $23.32 \pm 12.23 \mathrm{~Hz}$ (95\% CI: $20.58 \mathrm{~Hz}, 26.06 \mathrm{~Hz}$ ). For the $91 \mathrm{CHL}$ lesions, the mean size was $3.37 \pm 2.03 \mathrm{~cm}$ (range from $0.89 \mathrm{~cm}$ to $9.34 \mathrm{~cm}$ ) and the mean $\mathrm{R}_{2}$ * value was $3.66 \pm 2.37 \mathrm{~Hz}(95 \% \mathrm{CI}: 3.17 \mathrm{~Hz}, 4.15 \mathrm{~Hz})$. No significant differences in lesion size were found between the two groups of patients (students' $t$-test, $p<0.001$ ). Table 1 lists the $\mathrm{R}_{2} *$ results of the Mann-Whitney non-parametric $U$-test. The mean $\mathrm{R}_{2}$ * value of $\mathrm{HCCs}(\mathrm{Hz})$ was significantly higher than that of
CHLs $(p<0.001)$. The $\mathbf{R}_{2} *$ value of HCCs and CHLs were plotted in box and whisker format respectively on figure 1 . The boxes showed the distance between the first and the third quartiles, with the median marked as a line, and the "whiskers" indicating the range. The ROC curve was shown in figure 2. The optimal $\mathrm{R}_{2} *$ value calculated from the ROC curve was $9.48 \mathrm{~Hz}$, which resulted in a sensitivity of $96.20 \%(76 / 79)$, specificity of $97.80 \%(89 / 91)$, PPV of $97.44 \%$ (76/78), NPV of $96.74 \%$ (89/92), and a diagnosis accuracy of $97.06 \%(165 / 170)$. Figure 3 shows the typical appearance of CHL and HCG on axial $\mathrm{R}_{2} *$ maps. Overlapping of these two types of hepatic lesions was found and five lesions (5/ $170,2.94 \%$ ) involved in this study. Applying the $\mathrm{R}_{2}$ * measurement threshold of $9.48 \mathrm{~Hz}$ as a parameter, 2 out of 91 CHLs (2/91, $2.20 \%$ ) were erroneously considered as HCCs, whereas three HCCs (3/79, 3.80\%) were erroneously considered as CHLs.

\section{Discussion}

Treatment options and the overall management for HCC and CHL are different. Hepatic resection or transplantation are the best treatment options currently available for HCGs [4], and interventional therapies such as transcatheter arterial chemoembolization (TACE), radio-frequency ablation and microwave ablation are the alternative steps for those not eligible for surgical treatment [19]. In contrast, CHL does not require surgical resection, with the exception of large symptomatic lesions, due to the low rate of complications [3]. Percutaneous liver biopsy is currently considered as the gold standard for assessing hepatic neoplasm; however, this invasive method is costly and somewhat risky, thereby limiting its availability as an option for many patients [4]. A correct diagnosis of HCG and CHL based on noninvasive or minimally-invasive imaging would be of great benefit in guiding subsequent treatment planning.

This study demonstrates that $\mathrm{R}_{2}{ }^{*}$ is a valuable tool for differentiating HCG from CHL. Using $9.48 \mathrm{~Hz}$ as the threshold, we found a $96.20 \%$ sensitivity, a $97.80 \%$ specificity, a $97.44 \%$ PPV, a $96.74 \%$ NPV, and a diagnosis accuracy of $97.06 \%$. This result shows better differentiation than all previously reported studies in literature. Moreover, with a total acquisition time of 21 seconds, this method is much faster than all other reported methods in similar studies. Chan et al. [10] used moderate $\mathrm{T}_{2^{-}}$ weighted imaging with a scan time of more than 2.5 minutes to differentiate benign and malignant hepatic lesions. They achieved a $77 \%$ specificity and $88 \% \mathrm{PPV}$ with a $\mathrm{T}_{2}$ relaxation time threshold of $<112 \mathrm{~ms}$ being indicative of malignant lesion. Soyer et al [13] differentiated CHL from untreated malignant hepatic neoplasms with free-breathing diffusion-weighted MRI with a scan time around 2 minutes. With two independent readers, they reported a sensitivity of $65.7 \% \sim 100 \%$, a specificity of $85.7 \% \sim 100 \%$ and an accuracy of $82.9 \% \sim 94.3 \%$ depending on which threshold was applied to the ADC values. It is noteworthy that in the discussed study, two subjects underwent unnecessary surgery because their hepatic lesions were unfortunately misdiag- 
nosed as HCG on conventional MRI. The $\mathrm{R}_{2} *$ value of these two cases was 7.77 and $9.19 \mathrm{~Hz}$ respectively, which are smaller than our threshold value of $9.48 \mathrm{~Hz}$. In other words, they would have been correctly diagnosed if the usefulness of $R_{2}{ }^{*}$ was discovered earlier. In the present study, 2 out of 91 CHLs were also erroneously considered as HCGs, however, these two CHLs were characterized by typical imaging features on routine MRI. This study supports that $\mathrm{R}_{2}{ }^{*}$ values could potentially provide an effective quantitative parameter to help differentiate difficult or confusing HCC and CHL cases.

This study indicates that the mean $\mathrm{R}_{2} *$ value of $\mathrm{HCC}$ is significantly higher than that of CHLs. The exact mechanism behind this observation is unknown. A possible explanation is the lack of sufficient oxygenation in HCC. Hypoxia is common within a tumor micro environment due to the imbalance between oxygen supply from abnormal tumor vasculature and the high resource demand necessary for the rapid proliferation of tumor cells [1921]. It is well known that $R_{2} *$ is related to the oxygenation state of hemoglobin $[14,15]$. Blood in the microvasculature of HCG is lower in oxygenation and, accordingly, the deoxyhemoglobin concentration is higher which results in an increase in $R_{2} *$ values. CHL, on the other hand, involves a vascular malformation of the liver arterioles. It has been observed that the veins which drain the CHL, the peripheral branches of the portal vein, have $85 \%$ oxyhemoglobin saturation $[22,23]$. This increased concentration of oxyhemoglobin in CHLs can therefore result in a decrease in $\mathrm{R}_{2} *$ values. Furthermore, intra-tumor hemorrhage in HCCs, which is rarely encountered in CHLs, may also contribute to its increased $\mathrm{R}_{2} *$ value. Although $\mathrm{R}_{2} *$ values were obtained from

\section{References}

1. Ferlay J, Shin HR, Bray F, Forman D, Mathers C, et al. (2010) Estimates of worldwide burden of cancer in 2008: GLOBOCAN 2008. Int J Cancer 127: 2893-2917.

2. Hamilton SR, Aaltonen LA (2000) Pathology and genetics of tumors of the digestive system, world health organization classification of tumors, IARC Press: lyon. $314 \mathrm{p}$

3. Vilgrain V, Boulos L, Vullierme MP, Denys A, Terris B, et al. (2000) Imaging of atypical hemangiomas of the liver with pathologic correlation. Radiographics 20: 379-97.

4. Song TJ, Ip EW, Fong Y (2004) Hepatocellular carcinoma: current surgical management. Gastroenterology 127: S248-S260.

5. Lee JH, Lee JM, Kim SJ, Baek JH, Yun SH, et al. (2012) Enhancement patterns of hepatocellular carcinomas on multiphasicmultidetector row CT: comparison with pathological differentiation. Br J Radiol 85: e573-83.

6. Quinn SF, Benjamin GG (1992) Hepatic cavernous hemangiomas: simple diagnostic sign with dynamic bolus CT. Radiology 182: 545-548.

7. Leslie DF, Johnson CD, Johnson CM, Ilstrup DM, Harmsen WS (1995) Distinction between cavernous hemangiomas of the liver and hepatic metastases on CT: value of contrast enhancement patterns. AJR Am J Roentgenol 164: 625-629.

8. Ito K, Mitchell DG, Outwater EK, SzklarukJ, Sadek AG (1997) Hepatic lesions: discrimination of nonsolid, benign lesions from solid, malignant lesions with heavily $\mathrm{T}_{2}$-weighted fast spin-echo MR imaging. Radiology 204: 729-737.

9. Farraher SW, Jara H, Chang KJ, Ozonoff A, Soto JA (2006) Differentiation of hepatocellular carcinoma and hepatic metastasis from cysts and hemangiomas with calculated $T_{2}$ relaxation times and the $T_{1} / T_{2}$ relaxation times ratio. J Magn Reson Imaging 24: 1333-41.

10. Chan YL, Lee SF, Yu SC, Lai P, Ching AS (2002) Hepatic malignant tumour versus cavernous haemangioma: differentiation on multiple breath-hold turbo spin-echo MRI sequences with different $\mathrm{T}_{2}$-weighting and $\mathrm{T}_{2}$-relaxation time measurements on a single slice multi-echo sequence. Clin Radiol 57: 250-7.

11. Cieszanowski A, Szeszkowski W, Golebiowski M, Bielecki DK, Grodzicki M, et al. (2002) Discrimination of benign from malignant hepatic lesions based on their T2-relaxation times calculated from moderately $\mathrm{T}_{2}$-weighted turbo $\mathrm{SE}$ sequence. Eur Radiol 12: 2273-9.

12. Santoro L, Grazioli L, Filippone A, Grassedonio E, Belli G, et al. (2009) Resovist enhanced MR imaging of the liver: does quantitative assessment help in focal lesion classification and characterization? J Magn Reson Imaging 30: 1012-20.

13. Soyer P, Corno L, Boudiaf M, Aout M, Sirol M, et al. (2011) Differentiation between cavernous hemangiomas and untreated malignant neoplasms of the liver with free-breathing diffusion-weighted MR imaging: comparison with $\mathrm{T}_{2}$ weighted fast spin-echo MR imaging. Eur J Radiol 80: 316-24.
ROIs which did not include any visible hemorrhage on $\mathrm{T}_{1} \mathrm{WI}$ and $\mathrm{T}_{2} \mathrm{WI}$ in HCC, it does not mean that the ROI does not include micro-hemorrhage As demonstrated in recent studies [24,25], more intra-tumorous hemorrhage, especially micro-hemorrhage, were detected in $\mathrm{HCC}$ with susceptibility weighted imaging than with conventional $\mathrm{T}_{1} \mathrm{~W}$ and $\mathrm{T}_{2} \mathrm{~W}$ images. When there was internal spontaneous micro-hemorrhages, the extravasated hemoglobin within the tumor was converted to deoxyhemoglobin or hemosiderin. These paramagnetic substances could lead to local magnetic field inhomogeneity, resulting in the dephasing of protons and an increase in $\mathrm{R}_{2} *$ values [26,27].

This study has some limitations. The major weakness is the relatively small number of patients with histopathological confirmation of CHLs. This study mainly characterized CHL based on imaging characteristics and stability in size over time. To circumvent this limitation, only patients with histopathologically or clinically proven CHL as determined by two experienced radiologists after at least six months of CT and/or MRI follow-ups were selected.

In conclusion, this study demonstrates that using $\mathrm{R}_{2} *$ measurements from multi-echo $\mathrm{T}_{2}{ }^{*}$-weighted imaging appears to be a simple yet robust method to differentiate HCC from CHL with satisfying sensitivity and specificity.

\section{Author Contributions}

Conceived and designed the experiments: MS AL HW QS. Performed the experiments: QS DN BX QW. Analyzed the data: SW MS. Wrote the paper: MS SW AL ZW.

14. Jhaveri KS, Cleary SP, Fischer S, Haider MA, Pargoankar V, et al. (2013) Blood oxygen level-dependent liver MRI: Can It predict microvascular invasion in HCG? J Magn Reson Imaging 37: 692-9.

15. Yu DX, Ma XX, Zhang XM, Hou JW, Li CF (2010) Evaluation of blood oxygen level in hepatocellular carcinoma with noninvasive magnetic resonance multi-echo $\mathrm{R}_{2}{ }^{*}$ technique and its clinical significance. Zhonghua Yi Xue Za Zhi 90: $1463-6$.

16. Hardie AD, Romano PB (2010) The use of $\mathrm{T}_{2}{ }^{*}$-weighted multi-echo GRE imaging as a novel method to diagnose hepatocellular carcinoma compared with gadolinium-enhanced MRI: a feasibility study. Magn Reson Imaging 28: 281-5.

17. Hardie AD, Nance JW, Boulter DJ, Kizziah MK (2011) Assessment of the diagnostic accuracy of $\mathrm{T}_{2}{ }^{*}$-weighted $\mathrm{MR}$ imaging for identifying hepatocellular carcinoma with liver explant correlation. Eur J Radiol 80: e249-52.

18. Semelka RC, Brown ED, Ascher SM, Patt RH, Bagley AS, et al. (1994) Hepatic hemangiomas: a multi-institutional study of appearance on $\mathrm{T}_{2}$-weighted and serial gadoliniumenhanced gradient-echo MR images. Radiology 192: 401-6.

19. Dewhirst MW, Cao Y, Moeller B (2008) Cycling hypoxia and free radicals regulate angiogenesis and radiotherapy response. Nat Rev Cancer 8: 425-437.

20. Vaupel P, Harrison L (2004) Tumor hypoxia: causative factors, compensatory mechanisms, and Cellular response. Oncologist (Suppl 5): 4-9.

21. Overgaard J (2007) Hypoxic radiosensitization: adored and ignored. J Clin Oncol 25: 4066-74

22. Li GW, Chen QL, Jiang JT, Zhao ZR (2003) The origin of blood supply for cavernous hemangioma of the liver. Hepatobiliary Pancreat Dis Int 2: 367-70.

23. Sun XL, Li GW, Yang SY, Liu QF, Wang ZL, et al. (2006) Ultramicrostructure of hepatic cavernous hemangioma and its correlation with clinical characteristics. Shijie Hua Ren Xiao Hua Za Zhi 14: 3107-10.

24. Li RK, Zeng MS, Rao SX, Oiang JW, Dai YM, et al. (2012) Using a 2D multibreath-hold susceptibility-weighted imaging to visualize intratumoral hemorrhage of hepatocellular carcinoma at $3 \mathrm{~T}$ MRI: correlation with pathology. J Magn Reson Imaging 36: 900-6.

25. Tao R, Zhang J, Dai Y, You Z, Fan Y, et al. (2012) Characterizing hepatocellular carcinoma using multi-breath-hold two-dimensional susceptibility-weighted imaging: comparison to conventional liver MRI. Clin Radiol 67: e91-7.

26. Guo LF, Wang G, Zhu XY, Liu C, Cui L (2013) Comparison of ESWAN, SWISPGR, and 2D $\mathrm{T}_{2}{ }^{*}$-Weighted GRE Sequence for Depicting Cerebral Microbleeds. Clin Neuroradiol 23: 121-7.

27. Sehgal V, Delproposto Z, Haddar D, Haacke EM, Sloan AE, et al. (2006) Susceptibility-weighted imaging to visualize blood products and improve tumor contrast in the study of brain masses. J Magn Reson Imaging 24: 41-51. 